\title{
Undersampled Dynamic MRI Reconstruction by Double Sparse Spatiotemporal Dictionary
}

\author{
Juerong $\mathrm{Wu}^{1,2}$, Dongxiao $\mathrm{Li}^{1,2(\bowtie)}$, Xiaotian Qiao ${ }^{1}$, \\ Lianghao Wang $^{1,2}$, and Ming Zhang ${ }^{1,2}$ \\ ${ }^{1}$ Department of Information Science and Electronic Engineering, \\ Zhejiang University, Hangzhou 310027, China \\ lidx@zju.edu.cn \\ 2 Zhejiang Provincial Key Laboratory of Information Network Technology, \\ Hangzhou 310027, China
}

\begin{abstract}
Dynamic magnetic resonance imaging (dMRI) is widely used in human motion organ and functional imaging. But it requires reducing the imaging time to obtain high spatial and temporal resolution. This paper proposes a double sparse spatiotemporal dictionary model for compressed sensing reconstruction of dMRI from undersampled data. The model extends the ordinary 2-D dictionary to 3-D spatiotemporal dictionary by sparse representation of both the signals and dictionary atoms. Specifically, the first level sparse representation of dictionary atoms is learned with K-SVD algorithm. The second level sparse representation of spatiotemporal patches is obtained by OMP algorithm. An alternate iterative optimization is applied to solve the problem. Experiment results demonstrate that comparing with the state of the art method k-t FOCUSS and single level dictionary learning - DLMRI, the proposed method performs better in removing aliasing artifacts and in capturing temporal variations as well.
\end{abstract}

Keywords: Dynamic magnetic resonance imaging (dMRI) - Compressed sensing $(\mathrm{CS}) \cdot$ Double sparse spatiotemporal dictionary

\section{Introduction}

Dynamic magnetic resonance imaging (dMRI) plays an important role in clinical diagnoses, such as cardiac cine, perfusion and functional MRI. In order to capture the subtle changes in vivo, high spatial and temporal resolution is required in dMRI. But due to physical and physiological constraints, the motion during the full data acquisition process for one frame cannot be neglected, which leads to a loss of data consistency. To reduce the scan time, a range of techniques has been proposed, such as parallel imaging, fast-scan trajectory (spiral, radial) and randomly under sampling of k-space data [1]. However, they all led to ill-posed inverse problems of reconstruction since they do not satisfy the Nyquist sampling theorem.

The recent theory of compressed sensing (CS) aims to reconstruct signals and images from significantly fewer measurements and is thus suitable for dMRI 
reconstruction [2]. For example, k-t FOCUSS [3] successfully employed the compressed sensing theory for cardiac imaging applications by transforming the time varying image using Fourier transform along the spatial direction and residual encoding along the temporal direction. According to whether the reconstruction is processed in a transform domain or directly in the image domain, CS can be divided into two categories. The former has to find a sparse transform domain, such as DCT, wavelet [4], whichever meets the appropriate structure and fast numerical calculation demands. It is of low computational complexity but not flexible enough for the complex structure of images. The latter constructs a dictionary learned directly in image domain [5], and provides more flexible representation. However, its high degree of freedom demands a large amount of computation, which limits the practical size of the dictionary.

To deal with the existing problems mentioned above, we propose a new dictionary model that is flexible and of low complexity in computation. This work is inspired by the double sparse dictionary model proposed by Rubinstein et al. [6] which showed good performance in image denoising [7]. In under sampled MRI, the reconstruction quality mainly suffered from aliasing artifacts and image noise. And the temporal and spatial correlations in dynamic MRI make it very suitable to use a two-level dictionary. On the other hand, a lot of work has been done and demonstrated that dictionaries based on the image patch can preserve more local details than the global dictionaries [8-10].

A patch-based double sparse spatiotemporal DL model was developed in our work for under sampled dMRI reconstruction. First, the reconstructed image sequence is divided into overlapping patches along both spatial and temporal directions. Then we used a double sparse model to deal with the huge number of 3-D patches. Specifically, the first level sparse representation of dictionary atoms is trained using the K-SVD algorithm [11], which generalized the K-means clustering process and has been widely used in dictionary update stage. The second level sparse representation of spatiotemporal patches is obtained by orthogonal matching pursuit (OMP) algorithm [12], which is a greedy stepwise least squares sparse-coding algorithm. Last, alternate iterative optimization is applied to obtain the optimal sparse representation in two levels.

The rest of this paper is organized as follows. In Sect. 2, the basics of DL and double sparse dictionary model are briefly described. In Sect. 3, we will describe the proposed double sparse spatiotemporal DL model in detail. And alternate iterative optimization is used to solve the dMRI reconstruction problem. Section 4 presents the experiment results on cardiac cine imaging. We conclude our work in Sect. 5 and discuss future directions.

\section{Related Work}

Dictionary Learning (DL) focuses on building a suitable dictionary that provides efficient sparse representations for a class of signals [5]. Mathematically, given a set of signals $\mathrm{X}=\left\{x_{i}\right\}_{i=1}^{\mathrm{N}}$, DL aims to solve the following problem: 


$$
\min _{D, A}\|\mathrm{X}-\mathbf{D A}\|_{F} \text { s.t }\left\|\alpha_{i}\right\|_{0} \leq K, \forall i
$$

where $\|\bullet\|_{F}$ denotes the Frobenius norm, $\|\bullet\|_{0}$ is the $l_{0}$ norm, $\|\bullet\|_{2}$ is the $l_{2}$ norm, D stands for the overcomplete dictionary, $\mathrm{A}=\left[\alpha_{1}, \alpha_{2}, \ldots, \alpha_{\mathrm{N}}\right], \alpha_{i}$ is the sparse representation of $x_{i}$ over $\mathrm{D}$, and $K$ is the sparse constraint.

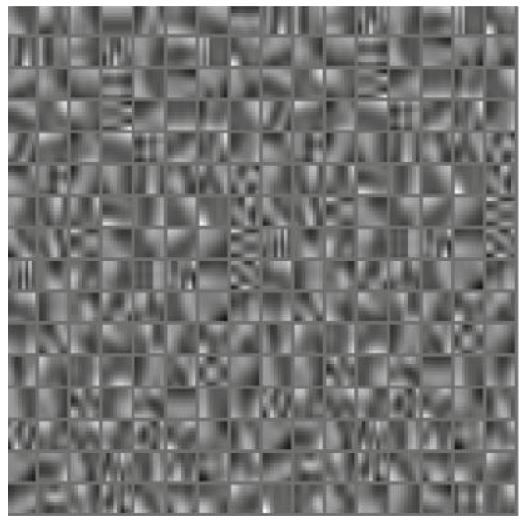

(a)

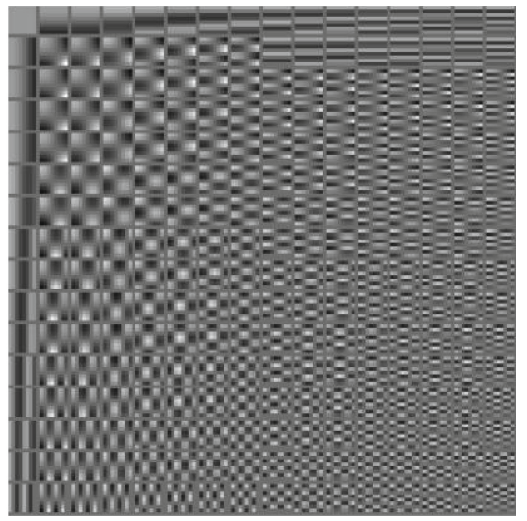

(b)

Fig. 1. (a) The trained dictionary atoms $(8 \times 8$ image patches). (b) 2-D DCT dictionary.

The double sparse dictionary model suggests that not only each signal can be represented as a sparse combination of atoms in the dictionary, but each atom of the dictionary has itself a sparse representation over some base dictionary [6]. Figure 1(a) shows an example of the first level overcomplete dictionary atoms. As we can see, these atoms are similar and regular patches, and can be efficiently represented by sparse coefficients with the second level DCT dictionary in Fig. 1(b). The double sparse dictionary is therefore expressed as

$$
\mathbf{D}=\Phi \mathrm{B}
$$

where B is the atom representation matrix, assumed to be sparse. Examples of base dictionary $\Phi$ include the discrete cosine transform (DCT) dictionary and Wavelet dictionaries. They are analytic dictionaries which have efficient implementations and strong structures.

Specifically, double sparse DL aims to solve the optimization problem

$$
\begin{array}{r}
\min _{B, A} \sum_{i j}\|\mathrm{X}-\Phi \mathrm{BA}\|_{F} \quad \text { s.t. } \forall i \quad\left\|\alpha_{i}\right\|_{0} \leq K \\
\text { s.t. } \forall j \quad\left\|\beta_{j}\right\|_{0} \leq L,\left\|\Phi \beta_{j}\right\|_{2}=1
\end{array}
$$


with $K$ as the sparse constraint of signals and $L$ as the sparse constraint of dictionary atoms. Note that in this formulation, the atom normalization constraint is commonly added for convenience.

\section{The Proposed Method}

\subsection{Patch-Based Spatiotemporal Dictionary}

To sparsely represent static images using DL, an image is divided into overlapping patches since patch based dictionaries can capture local image features effectively [8]. In dynamic MRI, we can also reconstruct the image sequence frame by frame in the same manner. But high coherence exhibits in temporal direction as well, which enables a sparse representation in both spatial and temporal domains [13]. We captured the consecutive patches in temporal direction as three dimensional spatiotemporal patches.

Let $R_{i j k}$ be the operator that extracts a spatiotemporal patch $x_{i j k}$ from dMRI image sequence $\mathrm{X}$, which is located at $(i, j, k)$ and with a predefined size of $\left(n_{f}, n_{p}, n_{t}\right)$. The corresponding DL problem is modeled as

$$
\begin{array}{r}
\min _{B, A} \sum_{i j k}\left\|\mathbf{R}_{i j k} \mathrm{X}-\Phi \mathrm{BA}\right\|_{F} \\
\text { s.t. }\left\|\alpha_{i j k}\right\|_{0} \leq K, \\
\left\|\beta_{i j k}\right\|_{0} \leq L,\left\|\Phi \beta_{i j k}\right\|_{2}=1, \forall i, j, k
\end{array}
$$

Here, $\mathrm{A}$ and $\mathrm{B}$ denotes the set containing all $\alpha_{i j k}$ and $\beta_{i j k}$ respectively. The base dictionary $\Phi$ is the Kronecker product of three 1-D DCT dictionaries. Assuming $\Phi_{0}$ is the DCT dictionary of 1-D signals, the 3-D spatiotemporal DCT dictionary is $\Phi=\Phi_{0} \otimes \Phi_{0} \otimes \Phi_{0}$. Generalizations to higher dimensions are straightforward to derive in the same way. So the two-level dictionary is more suitable for high dimensional reconstruction in MRI.

For the dMRI image sequence, a straightforward method is to build a single dictionary over all the frames, but it is only suitable for short period image sequence. The spatial structures and temporal variations would significantly change over a long period, thus calls for temporal dependent dictionaries. Specifically, we extracted a sub image sequence $\mathrm{X}_{\Omega}$ to construct the corresponding dictionary $\mathbf{D}_{\Omega}$ for this period. It reduces the dictionary size and adapts to the change of spatial structures and temporal variations.

\subsection{Dynamic MRI Reconstruction}

The problem formulation for dynamic MRI reconstruction based on double sparse dictionary learning needs to consider the following characteristics. First, it should be able to enforce the sparsity of the patches of the reconstructed image sequence in an adaptive dictionary and the sparsity of this dictionary's atoms in a base dictionary. Then, It should also be able to reduce the artifacts typically seen in the zero-filled (filled 
with zeros in the missing undersampled k-space data) Fourier reconstruction. At the same time, it should produce a reconstruction that is consistent with the acquired k-space data. Thus, based on the above double sparse spatiotemporal DL model, we formulate the dMRI reconstruction problem as follows:

$$
\begin{array}{r}
\min _{X, B, A} \sum_{i j k}\left\|\mathbf{R}_{i j k} \mathrm{X}-\Phi \mathrm{BA}\right\|_{F}+\lambda\left\|\mathbf{F}_{u} \mathrm{X}-\Upsilon\right\|_{F} \\
\text { s.t. }\left\|\alpha_{i j k}\right\|_{0} \leq K, \\
\left\|\beta_{i j k}\right\|_{0} \leq L,\left\|\Phi \beta_{i j k}\right\|_{2}=1, \forall i, j, k
\end{array}
$$

where $\mathrm{Y}$ is the undersampled $\mathrm{k}$-space data of $\mathrm{X}, \mathrm{F}_{\mathrm{u}}$ denotes the corresponding Fourier encoding matrix. The first term in the cost function corresponds to the quality of the double sparse representation of spatiotemporal patches with base dictionary $\Phi$. The second term in the cost enforces data fidelity in k-space, since the k-space measurements may be inaccurate and with noise. The weight $\lambda$ in the formulation is a tuning parameter, which depends on the measurement noise.

We used an alternating optimization procedure to solve the problem. First, $\mathrm{X}$ is assumed fixed, the sparse representation B of dictionary atoms and sparse representation A of signals are jointly learned. The K-SVD algorithm [11] is used to learn the dictionary D. Since the base dictionary $\Phi$ is set as 3-D DCT dictionary, the K-SVD algorithm mainly get the sparse representation B. Once the dictionary is learnt, the orthogonal matching pursuit (OMP) algorithm is used to find the sparse coding A [12]. Then, the double sparse representation $\mathrm{A}$ and $\mathrm{B}$ are fixed, and $\mathrm{X}$ is updated to satisfy data consistency. The least squares solution satisfies the normal equation

$$
\left(\sum_{i j k} \mathbf{R}_{i j k}^{H} \mathbf{R}_{i j k}+\lambda \mathbf{F}_{u}^{H} \mathbf{F}_{u}\right) \mathrm{X}=\sum_{i j k} \mathbf{R}_{i j k}^{H} \Phi \mathrm{B} \alpha_{i j k}+\lambda \mathbf{F}_{u}^{H} \Upsilon
$$

The superscript $H$ denotes the Hermitian transpose operation. $\mathbf{F}_{u}^{H} \Upsilon$ is the zero-filled reconstruction of the measured data. Solving (6) directly is impractical because it involves the inverse operation of a huge matrix. $\mathbf{F}_{u}^{H} \mathbf{F}_{u}$ is a $\mathrm{Q} \times \mathrm{Q}$ matrix and typically $\mathrm{Q}$ may be $256 \times 256$. We do some simplifications according to the properties of some parameters. The term $\sum_{i j k} \mathbf{R}_{i j k}^{H} \mathbf{R}_{i j k}$ is a diagonal matrix, equal to $\eta I$, where $\eta$ is a scaling factor equal to the size of patch $\left(n_{f} \times n_{p} \times n_{t}\right)$. And the huge matrix is converted to $\mathbf{F F}_{u}^{H} \mathbf{F}_{u} \mathbf{F}^{H}$, which is also a diagonal matrix with ones and zeroes [8]. We define the "patch averaged result", which means each pixel value obtained by averaging contribution of patches that cover it

$$
x_{p}=\frac{\sum_{i j k} \mathbf{R}_{i j k}^{H} \Phi \mathrm{B} \alpha_{i j k}}{\eta}
$$


Then we transform from image space to Fourier space and use the sampled subset of k-space to replace corresponding points. That ensures the reconstruction result is consistent with the undersampled k-space data. Finally, the reconstruction $\mathrm{X}$ is obtained by Inverse Fast Fourier Transform.

$$
x=x_{p}+\left(\frac{\lambda}{\lambda+1}\right) \mathbf{F}_{u}^{H}\left(y-\mathbf{F}_{u} x_{p}\right)
$$

Algorithm 1 shows the pseudo code. The algorithm is initialized with a zero-filled Fourier reconstruction, $\mathbf{F}_{u}^{H} \mathbf{Y}$. The stopping criterion of the algorithm is that a predefined maximum iteration number is reached.

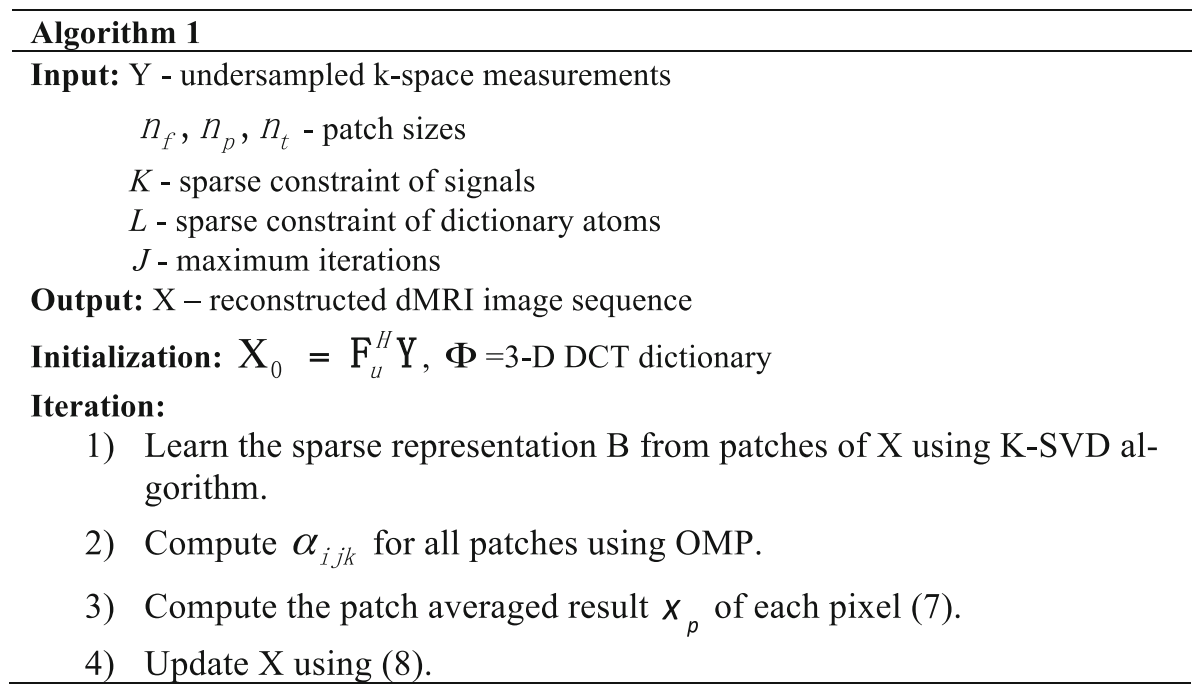

\section{Experimental Results}

We used two sets of dynamic cardiac cine data to validate the proposed method. The sizes of data sets are $256 \times 256 \times 25$ [3] and $190 \times 90 \times 70$ [14] (\#PE $\times \#$ FE $\times$ frame) respectively. Sampling schemes used in the experiment is Cartesian sampling with random phase encodes. The central part of the k-space is fully sampled with eight PE lines. The undersampling factor (the number of the fully sampled data points to that of the undersampled data point) in this experiment is 4 . The sampling masks and cardiac cine image sequences are shown in Fig. 2.

The performances of the proposed method were evaluated in two ways. First, we compared the results with the state of the art method in dMRI reconstruction - k-t FOCUSS [3]. In k-t FOCUSS, a motion estimation/compensation (ME/MC) approach was proposed to exploit the correlation between the frames using motion vectors 


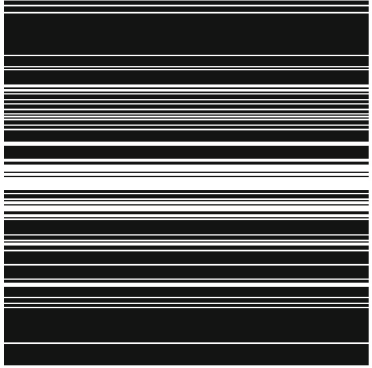

(a)

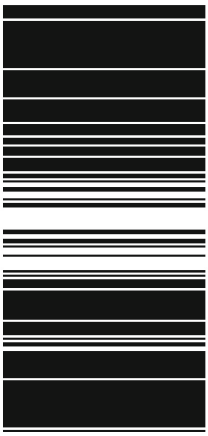

(c)

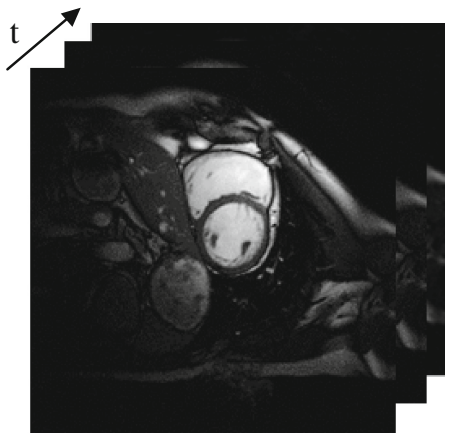

(b)

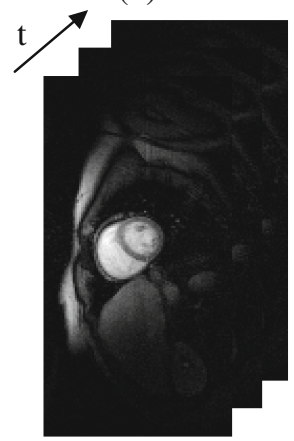

(d)

Fig. 2. (a) Sampling mask in k-space with 4 fold undersampling of dataset 1. (b) The reference dMRI image sequence of the cardiac cine in dataset 1. (c) Sampling mask of dataset 2. (d) The reference dMRI image sequence in dataset 2 .

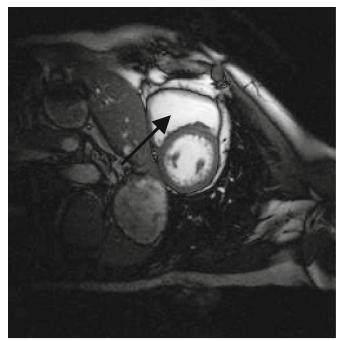

(a)

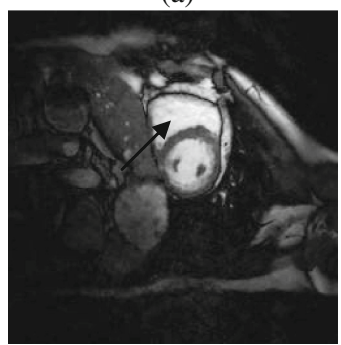

(d)

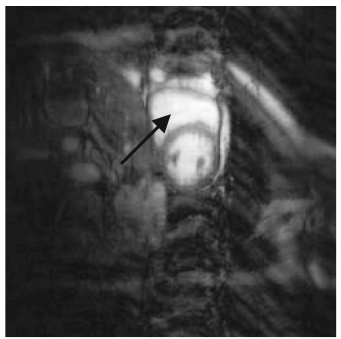

(b)

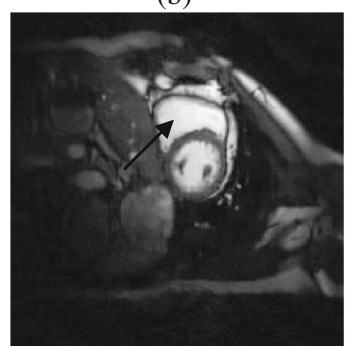

(e)

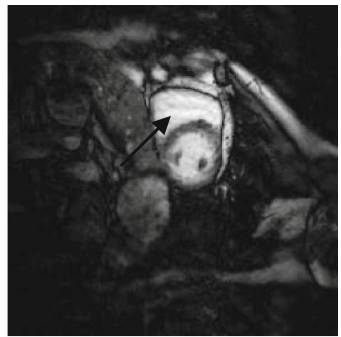

(c)

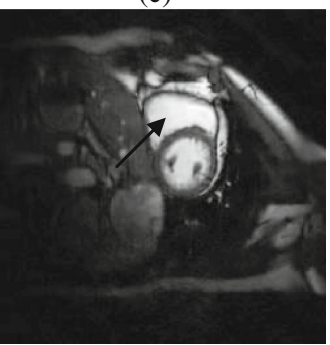

(f)

Fig. 3. Reconstructions of the fifteenth frame in dataset1. (a) Ground truth, (b) zero-filled, (c) k-t FOCUSS, (d) k-t FOCUSS with ME/MC, (e) DLMRI, (f) proposed method 
similar to video coding. We also compared with it to verify the effectiveness of the proposed model in capturing the high coherence in temporal direction. Second, we compared the results with DLMRI reconstruction frame by frame using 2-D patches [8], to see whether the two level double sparse dictionary performs better than ordinary single level dictionary.

In the experiments, the size of the 3D patch was $\left(n_{f}, n_{p}, n_{t}\right)=(6,6,6)$. Then the size of an atom $(\eta)$ was 216 and the atom number of 3-D DCT dictionary $\Phi$ was also 216.

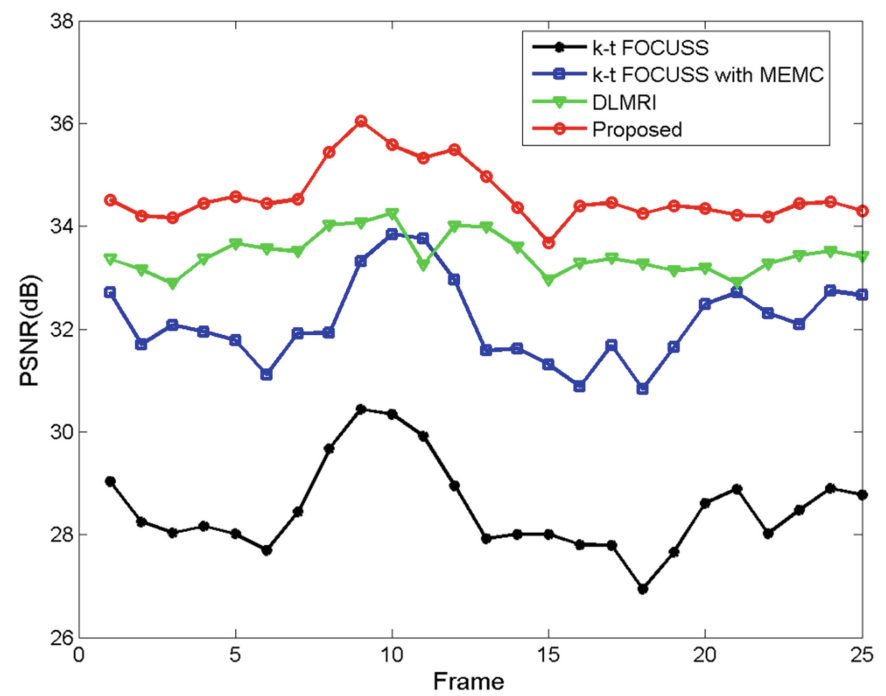

(a)

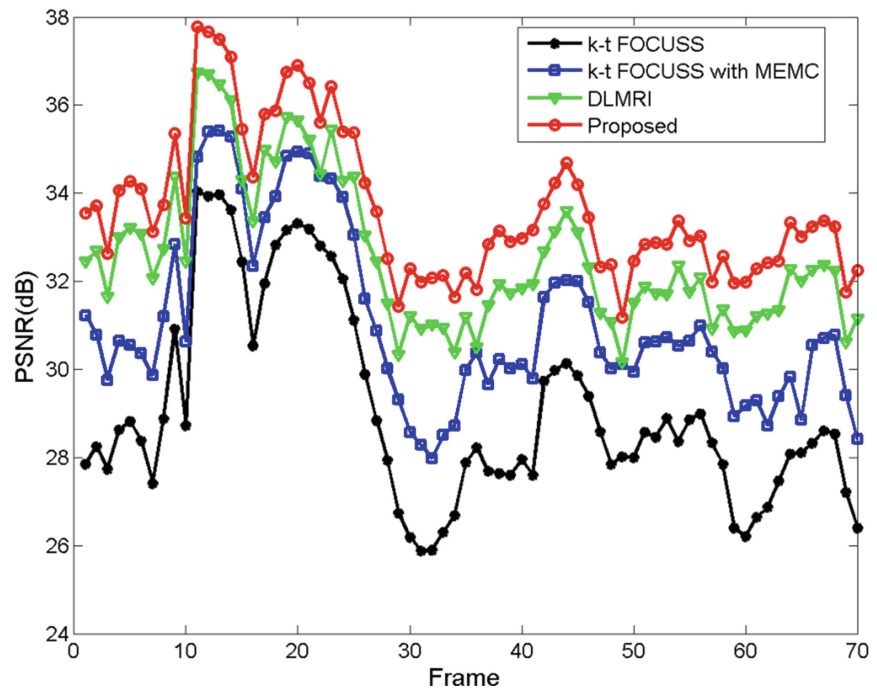

(b)

Fig. 4. Comparison of PSNR in different frames. (a) Dataset 1. (b) Dataset 2. 
We worked with maximum overlap of 3D spatiotemporal patch (the stride between corresponding pixel locations in adjacent image patches was 1). The sparse constraint of signals was set as $K=0.15 \times P \approx 32$ [8]. The sparse constraint of dictionary atoms $L$ was 6 . The maximum iterations $J$ was 10 .The relevant parameters in the 2-D DLMRI reconstruction experiment used the same setting for comparison.

Figure 3 shows the reconstruction results of the fifteenth frame in dataset 1 . The zero-filled reconstruction is badly blurred with artifacts. And the artifacts are also typically seen in k-t FOCUSS result. Although the quality of image is improved with ME/MC approach. But as the arrows indicate the details, the proposed method and DLMRI behave better in removing aliasing artifacts. It means that the patch-based dictionary learning method preserve more local detail than other optimization methods such as k-t FOCUSS.

The quality of the reconstruction is quantified using PSNR, the ratio of the peak intensity value of the reference to the mean-squared error (MSE) relative to the reference image. Figure 4 shows that the proposed method achieves a highest PSNR for all frames in both two datasets. This proves that with the double sparse design and high coherence in temporal direction, we had a better dictionary in dMRI reconstruction than 2-D MRI dictionary in the quality of image reconstruction. Otherwise, the two-level dictionary is easier to be extended to high dimensional reconstruction while get the same or even better reconstruction quality than single level dictionary.

In Fig. 5, we illustrated the temporal variation at a fixed position in the frequency encoding direction [15]. It can be observed that even zero-filled method captures main motion feature, but it is very blurry and lost some local contrasts. As the arrows point, the proposed method preserves more key motion details while others lost some temporal variations.

Figure 6 shows the reconstructions of the 43th frame by different methods and corresponding temporal profiles in dataset 2 . Also, the proposed method behaved better in removing aliasing artifacts and preserved more key motion details in temporal profiles than other methods.
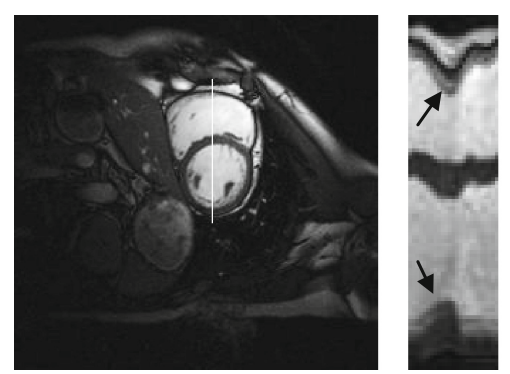

(a)

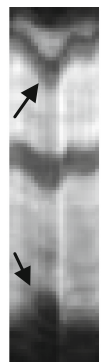

(b)

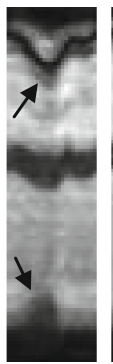

(c)

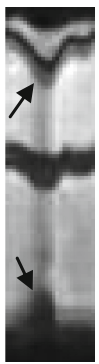

(d)

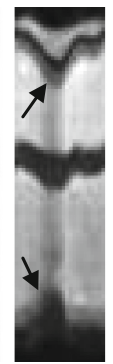

(e)

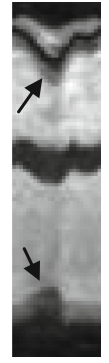

(f)

Fig. 5. Comparison of temporal profiles in dataset 1. (a) full sampled, (b) zero-filled, (c) k-t FOCUSS, (d) k-t FOCUSS with ME/MC, (e) DLMRI, (f) proposed method 

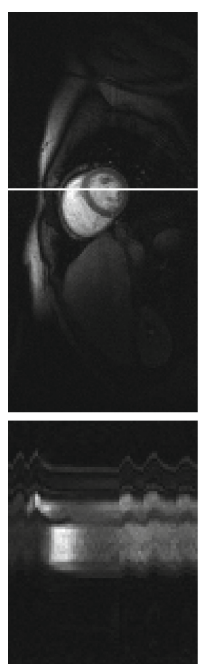

(a)

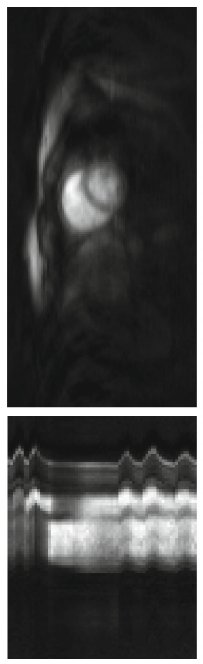

(b)
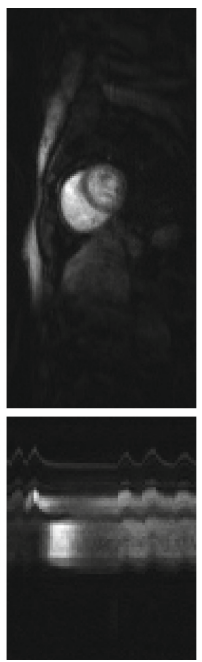

(c)
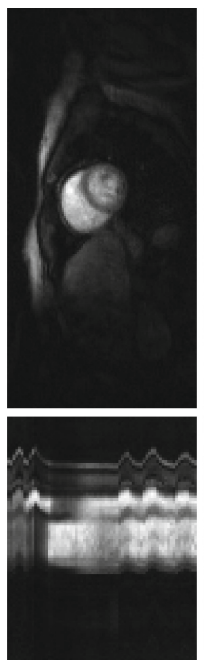

(d)

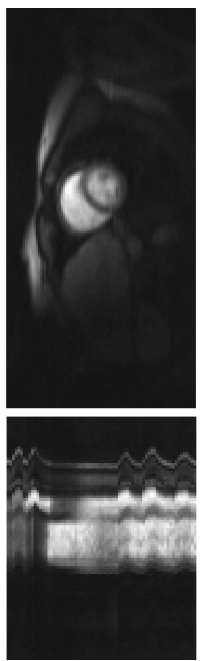

(e)
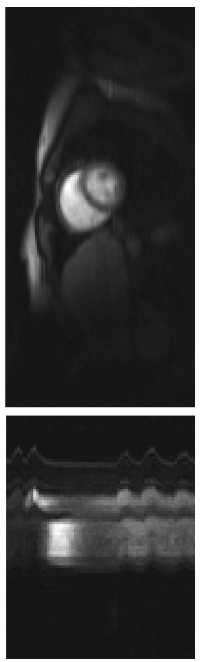

(f)

Fig. 6. Comparison of reconstructions of the 43th frame and temporal profiles in dataset 2. (a) full sampled, (b) zero-filled, (c) k-t FOCUSS, (d) k-t FOCUSS with ME/MC, (e) DLMRI, (f) proposed method

\section{Conclusion}

This paper proposed a patch-based double sparse DL model for dMRI reconstruction. This method combines the double sparse model and high coherence in spatial and temporal domains to train a 3-D dictionary, which is able to capture local structure spatially and temporally. The experimental results show that our method performs better in removing aliasing artifacts compared with k-t FOCUSS, a state of the art method in dMRI. And for the single level dictionary learning - DLMRI, the proposed method is easier to be extended to high dimensional space. In the future work, we would like to extend the model for 4-D dMRI reconstruction, i.e. reconstruction of 3D volumetric image sequence.

Acknowledgment. This work was supported in part by the National Natural Science Foundation of China (Grant No. 61271338, 61401390), the Zhejiang Provincial Natural Science Foundation of China (Grant No. LQ14F010005), and the Open Projects Program of National Laboratory of Pattern Recognition of China (Grant No. 201306308).

\section{References}

1. Yang, Z.H., Feng, F., Wang, X.Y.: A Guide to Technique of Magnetic Resonance Imaging: Criterion of Examination, Clinical Strategy and Application of New Techniques. People's Military Medical Press, Beijing (2007)

2. Gamper, U., Boesiger, P., Kozerke, S.: Compressed sensing in dynamic MRI. J. Magn. Reson. Med. 59(2), 365-373 (2008) 
3. Jung, H., Sung, K., Nayak, K.S.: k-t FOCUSS: a general compressed sensing framework for high resolution dynamic MRI. J. Magn. Reson. Med. 61(1), 103-116 (2009)

4. Lustig, M., Donoho, D., Pauly, J.M.: Sparse MRI: the application of compressed sensing for rapid MR imaging. J. Magn. Reson. Med. 58(6), 1182-1195 (2007)

5. Tosic, I., Pascal, F.: Dictionary learning. J. Signal Process. Mag. 28(2), 27-38 (2011)

6. Rubinstein, R., Zibulevsky, M., Elad, M.: Double sparsity: learning sparse dictionaries for sparse signal approximation. IEEE Trans. Signal Process. 58(3), 1553-1564 (2010)

7. Liang, R., Zhao, Z., Li, S.: Image denoising using learned dictionary based on double sparsity model. In: 2011 4th International Congress on Image and Signal Processing (CISP), vol. 2, pp. 691-695. IEEE (2011)

8. Ravishankar, S., Bresler, Y.: MR image reconstruction from highly undersampled k-space data by dictionary learning. IEEE Trans. Med. Imaging 30(5), 1028-1041 (2011)

9. Wang, Y., Ying, L.: Compressed sensing dynamic cardiac cine MRI using learned spatiotemporal dictionary. IEEE Trans. Biomed. Eng. 61(4), 1109-1120 (2014)

10. Awate, S.P., DiBella, E.V.R.: Spatiotemporal dictionary learning for undersampled dynamic MRI reconstruction via joint frame-based and dictionary-based sparsity. In: 2012 9th IEEE International Symposium on Biomedical Imaging (ISBI), pp. 318-321. IEEE (2012)

11. Aharon, M., Elad, M., Bruckstein, A.: K-SVD: an algorithm for designing overcomplete dictionaries for sparse representation. IEEE Trans. Signal Process. 54(11), 4311-4322 (2006)

12. Pati, Y.C., Rezaiifar, R., Krishnaprasad, P.S.: Orthogonal matching pursuit: Recursive function approximation with applications to wavelet decomposition. In: Signals, Systems and Computers, pp. 40-44 (1993)

13. Wang, Y., Zhou, Y., Ying, L.: Undersampled dynamic magnetic resonance imaging using patch-based spatiotemporal dictionaries. In: 2013 IEEE 10th International Symposium on Biomedical Imaging (ISBI), pp. 294-297. IEEE (2013)

14. Lingala, S.G., Jacob, M.: Blind compressive sensing dynamic MRI. IEEE Trans. Med. Imaging 32(6), 1132-1145 (2013)

15. Wang, Y., Ying, L.: Compressed sensing dynamic cardiac cine MRI using learned spatiotemporal dictionary. IEEE Trans. Biomed. Eng. 61(4), 1109-1120 (2014) 